DOSSIERS BIBLIOGRAPHIQUES

\title{
HISTOIRE GÉNÉRALE
}

John O'Malley, The First Jesuits. Cambridge, MA/Londres, Harvard University Press, 1993. $16 \times 23,457$ p., index.

Les Jésuites à l'âge baroque, 1540-1640. Éd. Luce GIARD et Louis DE VAUCELLES. Grenoble, Jérôme Millon, 1996. 16 × 23, 291 p., index (Histoire des jésuites de la Renaissance aux Lumières).

The Jesuits. Cultures, sciences, and the arts, 1540-1773. Éd. John O'MALLEY, Gauvin A. Bayley, Steven J. Harris et T. Frank Kennedy. Toronto/Buffalo/ Londres, University of Toronto Press, 1999. $15 \times 23,716 \mathrm{p}$.

Dans un ensemble consacré au renouveau de l'historiographie de la Compagnie de Jésus, et à ce que l'introduction de cet ensemble qualifiait de moment " généraliste ", au-delà des distinctions catégorielles en usage dans la production institutionnelle passée et au seuil de nouvelles formes de spécialisation historiennes (les unes et les autres pouvant d'ailleurs s'être combinées dans le passé... et se retrouver dans l'avenir), il était évidemment difficile de ne pas réserver une synthèse bibliographique à l'histoire générale de l'Ordre dans les publications récentes. Mais il est tout aussi difficile d'honorer cet engagement : car le renouveau entraîne déjà irrésistiblement une conception thématique de cette histoire. Les différents - et multiples - domaines dans lesquels intervient, d'une manière ou d'une autre, l'activité des jésuites se trouvent placés au centre, et les « grands récits » traditionnels sur la naissance, la croissance, la maturité, les déclins et les renaissances de la Compagnie se trouvent éclatés dans une littérature qui ne relève plus du genre narratif, ni, comme nous venons de le rappeler (sans préjuger des lendemains), ne relaie des choix spécifiquement liés aux finalités manifestes de l'institution: apostolat, évangélisation, conversion, catéchisation, etc. Du coup, les ouvrages généraux récents pourraient assez facilement être distribués entre les synthèses qui suivent. Il nous a semblé cependant que trois de ces ouvrages devaient être considérés pour eux-mêmes, non seulement en raison de la qualité de leur conception (surtout dans le cas, toujours difficile, des publications collectives), mais aussi parce qu'ils représentaient chacun une tendance particulière.

On pourrait dire, en forçant un peu le trait, que The First Jesuits de John O'Malley ${ }^{1}$ s'efforcerait à une histoire organisée selon la logique du développement de

1. On trouvera dans ce même numéro, supra, p. 428-439, les réponses de John O'Malley aux questions que nous lui avons posées, ainsi qu'à Louis Châtellier, Dominique Julia et Luce Giard, également coéditrice des Jésuites à l'âge baroque.

Revue de synthèse : $4^{\mathrm{e}} \mathrm{S}$. $\mathrm{n}^{\text {os }}$ 2-3, avr.-sept. 1999, p. 433-439. 
l'institution, et pourrait revendiquer ainsi l'héritage des " grands récits »; que Les Jésuites à l'âge baroque, issu du colloque tenu en France, à Chantilly, en 1991, à l'occasion du quatre cent-cinquantième anniversaire de la fondation de la Compagnie (et du cinquième centenaire de la naissance d'Ignace de Loyola), illustrent le passage à une histoire centrifuge, inscrivant des jésuites particuliers dans des sphères hêtérogènes; alors que les actes, monumentaux, du récent colloque de Boston (1997), auxquels nous accorderons ici la plus grande place, explorent une troisième voie, la plus riche sans doute, la plus périlleuse aussi, celle d'une conception de la Compagnie de Jésus, dans sa globalité, comme observatoire d'une époque le premier âge moderne - au sein de laquelle son omniprésence n'a d'égal que son caractère polymorphe, selon le principe insaisissable et impérieux du modo nostro, qui hante, comme un chiffre, ce dernier recueil.

Dans The First Jesuits, O'Malley réussit le tour de force de réaliser la première histoire " totale " des premières décennies de l'Ordre depuis les grandes synthèses du XviI ${ }^{e}$ siècle. Prédication, confession, direction spirituelle, engagements caritatifs, enseignement, conquête intellectuelle (théologique, scientifique), positions politiques, entreprises missionnaires proches et lointaines - tout cela compose, selon son projet, une profonde immersion des membres du nouvel Ordre dans la vie du peuple chrétien, dans un large décentrement des seuls enjeux de la Contre-Réforme ecclésiastique romaine. Ce faisant, O'Malley, comme il le fera quelques années plus tard, dans le volume de 1999, en dédouanant Ignace de Loyola d'un contreréformisme dont il impute la promotion à Jérôme Nadal (ce qui n'est probablement pas dénué de fondement étant donné les implications directes de celui-ci dans le monde germanique), donne de la fondation de l'Ordre une vision discutable, en particulier pour ce qui concerne l'interprétation, qui reste largement à explorer malgré l'utile volume dirigé en 1994 par Mario Zanardi ${ }^{2}$, du séjour d'Ignace à Venise dans les années 1537-1538. Il reste que l'ouvrage développe toute une série d'analyses extrêmement fines et précises, malgré l'ampleur des sources mobilisées, sur l'engendrement de l'institution, en particulier à travers l'étude de l'élaboration des formes du vœu en usage dans la Compagnie. The First Jesuits restera sans doute, pour longtemps, une introduction indispensable à toute enquête sur cette période, non seulement pour ce qui concerne l'histoire de la Compagnie de Jésus, mais plus généralement pour toute approche dynamique de l'effervescence institutionnelle des premières décennies de l'Europe tridentine.

Les Jésuites à l'âge baroque est un livre difficile à résumer en bref en fonction même de son extrême ouverture (qui fut aussi une ouverture remarquable - et remarquée - de la Compagnie de Jésus elle-même, fruit d'une collaboration entre des historiens de l'Ordre et des chercheurs extérieurs). On devra donc se contenter ici d'en signaler - injustement sans doute - les articles les plus importants : ceux

2. I Gesuiti a Venezia. Momenti i problemi di storia veneziana della Compagnia di Gesu, éd. Mario Zanardi, Padoue, Gregoriana Libreria Editrice, 1994. Mario Zanardi, qui vient de disparaître, fut pour tous ceux qui eurent à fréquenter, ces dernières années, les Archives jésuites romaines, un guide ouvert et attentif. Nous tenions à lui rendre ici ce simple hommage. 
de Luce Giard sur les Constitutions, d'Adrien Demoustier sur les Exercices spirituels, de Louis Marin enfin - pour l'un de ses derniers textes - sur le Récit d'Ignace; l'étude approfondie de Dominique Julia, qui en est l'un des meilleurs connaisseurs, sur la genèse de la Ratio studiorum, de Bernard Dompnier sur les missions intérieures ${ }^{3}$, domaine insuffisamment exploré encore. On retiendra, enfin, les recherches politiques et théologiques de Robert Bireley, dans le prolongement de son ouvrage important sur le Prince chrétien ${ }^{4}$, de Christian Jouhaud, sur l'écriture polémique du jésuite français François Garasse, de Jean-François Courtine, enfin, sur l'œuvre de Francisco Suarez'.

On retrouve John O'Malley dans The Jesuits. Cultures, sciences and the arts, 1540-1773, dont il a coordonné la publication avec Gauvin A. Bailey, Steven J. Harris et T. Frank Kennedy. Du reste, cet ouvrage important, ne serait-ce que par son volume (32 articles) et la somme d'informations qu'il transmet (en particulier pour ce qui concerne la bibliographie la plus récente : les spécialistes de l'histoire missionnaire, par exemple, ne pourront pas ne pas faire de cet ouvrage un instrument de référence), est, nous semble-t-il, pour O'Malley, une sorte de somme et d'aboutissement, près de dix ans après un article marquant, publié en 1991, «Was Ignatius Loyola a church reformer? How to look at early modern catholicism ${ }^{6}$ ". Sa ligne de force était: les historiens de l'époque moderne doivent aujourd'hui, si l'on peut résumer les choses ainsi, accueillir les jésuites parmi les chrétiens en ouvrant grandes les portes des institutions, qu'il s'agisse de l'institution particulière de la Compagnie, de l'Église en général ou de son émanation essentielle de la période, à savoir le concile de Trente. Les jésuites sont des chrétiens avant d'être des ecclésiastiques. Il faut les rédecouvrir ainsi (comme The First Jesuits y réussit d'ailleurs avec beaucoup de force) en amont d'une longue histoire que O'Malley, dans l'importante

3. Les actes du colloque tenu en mars 1999 à Chambéry sur les missions intérieures françaises et italiennes du $\mathrm{XVI}^{\mathrm{e}}$ au $\mathrm{xIX}^{\mathrm{e}}$ siècle apporteront des éléments nouveaux. Voir, également, sur l'espace ibérique, supra p. 359-380.

4. Robert BIRELEY, The Counter-Reformation prince. Anti-Machiavellianism or catholic statecraft in early modern Europe, Chapel Hill/Londres, University of North Carolina Press, 1990.

5. Auquel il a consacré par ailleurs un livre capital, Suarez et le système de la métaphysique, Paris, Presses universitaires de France, 1990, qui apporte un éclairage décisif sur le rôle des jésuites dans l'acheminement d'une ontologie contemporaine des développements de la science moderne, livre tout récemment enrichi d'un recueil d'articles, Nature et empire de la Loi. Études suaréziennes, Paris, Vrin/École des hautes études en sciences sociales, 1999.

6. The Catholic Historical Review, vol. 57, 1991, p. 177-193. Il n'est sans doute pas indifférent de préciser que cet article était la reprise d'une conférence donnée par O'Malley à l'occasion de son élection à la présidence de l'American Catholic Historical Association : les circonstances de ce discours illustraient l'inscription de l'histoire des jésuites dans l'histoire du catholicisme moderne. C'est aussi, nous semble-t-il, le parcours qui conduit de cet article au colloque de Boston (dont O'Malley n'est évidemment pas le seul « auteur ", mais dont l'article de 1991 est réellement un point de départ), qui permet de comprendre le long retour de l'introduction de 1999 sur le problème taxinomique, historiographique et plus largement idéologique de la désignation d'une « Réforme ", d'une " Contre-Réforme ", d'une " Réforme catholique », etc. qui a perdu aujourd'hui de son mordant pour un grand nombre d'historiens de la période. On retrouve d'ailleurs cette même inflexion rétrospective dans l'article de Gauvin A. Bailey sur l'art jésuite, comme si, dans une époque charnière comme la nôtre, il importait de revenir sur ses pas avant de reprendre sa marche. 
introduction au volume de 1999, ouvre avec Jérôme Nadal, l'un des principaux collaborateurs d'Ignace et le premier théoricien de l'institution jésuite, et clôt avec Hubert Jedin, le grand historien du concile de Trente et « inventeur », à l'époque contemporaine, de la place de ce Concile dans l'histoire de l'époque moderne.

Les domaines de recherche personnels des quatre coordinateurs, jésuites, de ce volume, outre O'Malley - lui-même connu, tout à fait indépendamment de ses recherches sur les "premiers jésuites ", pour des travaux sur l'histoire culturelle de la Renaissance italienne -, nous fournissent une première indication précieuse, confirmée par les équilibres globaux du volume sur les grands apports, mais peutêtre aussi sur certaines limites (comme Luce Giard le note dans les " Réflexions " fructueuses et incisives qui referment le volume) de la mise en œuvre de l'histoire jésuite comme "fenêtre ${ }^{7}$ » sur le monde. Bailey et Kennedy s'intéressent à l'histoire des arts (visuels pour le premier, sonores pour le second), principalement sur le terrain missionnaire, Harris à l'histoire des sciences. L'intitulé des différentes parties parle de lui-même. En effet, les deux premières, « Repenser l'histoire jésuite » et "La scène romaine ", sont suivies de : " Mobilité. Missions outre-mer et circulation de la culture », « Rencontres de l'autre. Entre assimilation et domination », «Tradition, innovation, adaptation » et «Conversion et confirmation. La dévotion et les arts". Le texte liminaire du recueil déclare d'ailleurs : "Nous avons voulu mettre en lumière les aspects suivants : l'usage jésuite des arts dans l'évangélisation et la transmission de la foi et de la dévotion; la recherche scientifique dans les collèges et dans les missions, et sa relation à la foi; la théorie et la pratique des conditions de recevabilité du christianisme par les Philippins, les Chinois », etc. Ces orientations auraient pu être davantage réfléchies pour elles-mêmes; elles correspondent, certes, à une réalité de la recherche actuelle (cette livraison de la Revue de synthèse en prend acte, elle aussi, dans son ensemble), mais dans la mesure où les études américaines, en fonction même d'une autonomie réelle de l'historiographie jésuite outre-Atlantique, au sein même de la Compagnie, par rapport aux traditions de l'institution, dominent le paysage. Or c'est un paysage en mouvement, et ces orientations doivent venir au débat: car elles font de la "fenêtre * jésuite une fenêtre trop transparente, en contournant finalement l'institution elle-même, dans ses modes de reproduction (toute la question de la sélection et de la formation des individus serait ici en jeu, et avec elle une définition et une conception de ces indivi$\operatorname{dus}^{8}$ ) et dans ses liens avec d'autres institutions (où l'on retrouverait la question politique) ${ }^{9}$; ce que l'on appelle la spiritualité jésuite (à laquelle nous avons jus-

7. Pour reprendre l'expression utilisée par J. O'MALLEY, in The First Jesuits, p. 4.

8. L'article de Thomas V. CoHEN, Why the Jesuit joined? (conclusions d'une thèse toujours inédite), publié en 1974 par The Canadian Historical Association, reste aujourd'hui encore l'une des seules études consacrées à ces problèmes, à partir de l'exploitation (à poursuivre) d'un questionnaire diffusé par Jérôme Nadal dans les provinces d'Espagne au début des années 1560 .

9. On peut évoquer, au titre d'exemple complémentaire de ce contournement, l'édition française récente d'un choix d'œuvres de Jêrôme NADAL, Contemplatif dans l'action, Paris, Desclée De Brouwer, 1996 : toutes sont systématiquement amputées des développements concernant l'institution, et l'on ne retient que celles qui intéressent le « chrétien » en général. Or, il n'est pas plus institutionnel, précisément, qu'une démarche qui, expressément placée du point de vue de l'institution, privilégie la perspective externe. C'est l'institution elle-même qu'il importe de placer dans le champ de vision - quitte, probablement, à constater le mor- 
tement tenu ici à consacrer une synthèse bibliographique, malgré la relative rareté des travaux neufs) se trouve au croisement de ces deux axes : les Exercices spirituels ne sont-ils pas un instrument doublement décisif, de la transmission interne et du rayonnement externe de l'Ordre, et l'étude de leur pratique ne provoquerait-t-elle pas nécessairement la rencontre de l'exercice d'un pouvoir et de l'exercice d'une autorité, sur un terrain probablement très exposé, non seulement aux spectres de l'« antijésuitisme », mais aussi, plus profondément peut-être, à la menace d'une articulation, en forme de dispersion, sur d'autres circuits d'influence, d'autres logiques de relations? C'est pourtant bien aussi sur ce terrain que l'on découvrirait peut-être l'une des spécificités, tant recherchées, du modo nostro.

Les grands équilibres du volume sont dans la cohérence de ces choix fondamentaux et il faut souligner la réussite d'une mobilisation de savoirs impressionnante. On ne peut retenir ici que quelques-unes des étapes les plus remarquables de ce long parcours.

L'article de Bailey, déjà cité (note 6), propose un bilan complet des recherches sur l'«art jésuite » depuis le début du siècle, mais surtout bien sûr dans les deux dernières décennies, en particulier dans le monde missionnaire. L'auteur annonce d'ailleurs la publication prochaine de son ouvrage, A global partnership. Art on the jesuit mission in Asia and the Americas (1540-1773), qui sera certainement une somme sur le sujet, et pour la première fois une somme comparative, permettant de prendre la mesure de l'ampleur des variations, mais aussi, peut-être, et d'une manière moins attendue, des répétitions et des constantes. L'une des suggestions principales de cet article fouillé est, nous semble-t-il, dans son évolution : de l'art missionnaire mondial, Bailey enchaîne sur l'architecture (qui représente le noyau dur de la bibliographie actuelle), puis sur les architectes (à partir du constat que tous les architectes des jésuites n'étaient pas jésuites, et qu'inversement tous les architectes jésuites ne travaillaient pas pour la seule Compagnie), enfin sur les artistes en général, et sur l'exigence, qu'il pose pour l'avenir, d'une étude des trajectoires singulières. Depuis l'horizon mondial, et en fonction du « jeu » qu'il ouvre, on en vient aux individus singuliers, ultimes artisans de ce jeu. On observera, enfin, deux traits complémentaires de l'article de Bailey : le premier, quelque peu surprenant, est qu'il continue de transmettre le «mythe fondateur " de la commande, du commandement fait par Ignace de Loyola à Jérôme Nadal de composer le recueil des Evangelicae historiae imagines; le second, inverse, mais profondément solidaire de l'autre, est qu'il marginalise sans doute à l'excès, dans son combat contre la notion ancienne d'un « art jésuite », les questions liées à la corrélation, dans les dernières années du $\mathrm{XVI}^{\mathrm{e}}$ siècle, entre la première Compagnie et le développement du courant «antimaniériste ${ }^{10} »$.

cellement, l'hétérogénéité, les discontinuités de ce nouveau paysage. Mentionnons aussi, par rapport à cela, le contre-exemple (très rare aujourd'hui encore), d'un article de José Martin MilLán, "Transformación y crisis de la Compañia de Jesús (1578-1594) », in I religiosi a corte. Teologia, politica e diplomatia in Antico Regime, éd. Flavio Rurale, Rome, Bulzoni, 1998, qui inscrit un épisode interne, voire micro-politique, de l'histoire de la Compagnie de Jésus dans un contexte large.

10. Selon la formulation de Walter Friedlander, le grand historien du Caravage, dans son Maniérisme et anti-maniérisme dans la peinture italienne, $1^{\text {re }}$ éd. 1957, Paris, Gallimard, 1991. 
La deuxième partie du livre, la "Scène romaine ", avec une importante étude de Louise Rice sur les rituels pégagogiques (déclamations, disputes, " performances» en général) et la culture emblématique du Collège romain. Il aurait sans doute été intéressant (c'est une barrière disciplinaire qui reste à lever) de faire intervenir plus directement la référence théâtrale pour cerner la figure de l'élève du Collège, « image vivante » promu parallèlement à la réserve de la première culture jésuite sur l'usage sacré des images.

Les recherches de Michael John Gorman et de Margarete Murata sur Athanasius Kircher et le Collège romain sont elles aussi intéressantes, bien que les choix de l'ouvrage en général pèsent lourd ici : la Compagnie de Jésus comme " fenêtre " sur le monde, c'est aussi le Collège romain comme «vitrine" de la Compagnie. L'ambivalence des liens et des écarts entre le centre et la périphérie demanderait probablement d'autres développements : le Collège romain fut, certes, un modèle, mais fut-il un modèle à suivre?

La troisième partie combine avec force deux domaines de recherches heureusement réunis dans l'historiographie actuelle : l'histoire des missions et l'histoire des sciences, avec les articles de Florence C. Hsia (sur l'activité des jésuites mathématiciens en Chine au Xvir ${ }^{e}$ siècle) et de Steven Harris (sur la connaissance de la nature dans l'entreprise missionnaire, en Extrême-Orient aussi, selon un déséquilibre entre les deux Indes qui n'est pas tout à fait remis en cause ici, mais qui est, partiellement au moins, historiquement fondé par la conception même des missions dans les deux mondes). On trouve également dans cette partie, largement ouverte, comme la suivante d'ailleurs, aux nouveaux aspects de l'historiographie, un recentrage intéressant de Dominique Deslandres (non sans analogie avec l'approche, chez Bailey, de la singularité des artistes jésuites) sur la figure du missionnaire, à partir du constat de l'extrême imbrication des ordres religieux sur les terres de mission et de la relation entre missionnaire et missionné : non seulement le missionnaire projette sur la réalité des " autres" une catégorie de l'"Autre" - pour reprendre les termes de l'auteur - mais il s'identifie, en quelque sorte, à cette catégorie, en faisant de son propre destin comme de celui de l'«Autre » un instrument de la grâce. De ce fait, c'est moins du missionnaire jésuite qu'il s'agit de rendre compte que d'un individu dont les sources jésuites permettent, pour une part, de rendre compte, et qui ne se confond pas non plus avec son objet (l'Autre et son monde). Il semble qu'on puisse atteindre ici à un point d'équilibre entre une histoire internaliste de l'institution selon les termes utilement employés par Luce Giard dans ses « Réflexions » finales - et une histoire externaliste de ses terrains.

La quatrième partie, consacrée elle aussi à l'histoire des missions lointaines (les missions intérieures, en terre européenne, sont très peu représentées dans le volume), fait la démonstration de la maturité atteinte par les recherches sur l'apprentissage missionnaire des langues : on retiendra en particulier l'étude de Qiong Zhang sur la transformation jésuite des concepts de la pensée confucéenne, où l'on observe la constitution d'une troisième langue, ni celle de la tradition chinoise, ni celle de la tradition chrétienne, mais qui ouvre l'espace d'une double intelligence, ou d'un double malentendu. Il ne faut pas sous-évaluer ici, comme l'article de Nicolas Standaert y insiste avec vigueur, la force de l'altération à l'œuvre dans l'entreprise missionnaire : on ne peut imaginer convertir une culture comme celle de la Chine qu'en se convertissant à elle pour, dans ce passage, tenter de faire la preuve de sa converti- 
bilité $^{11}$. Il faut citer, enfin, l'article très riche de René $\mathrm{B}$. Javellana sur les Philippines. On y apprend, par exemple, comment les pères jésuites à l'article de la mort entendent la lecture d'une prière traduite par leurs soins dans la langue locale (ici le Tagalog) et prononcée par un groupe $d^{\prime}$ ' indigènes ", qui disent donc dans leur langue des mots que le mourant a appris dans la sienne. Il écoute d'abord, dans ce rite étrange, des mots dont il croit qu'ils sont entendus : c'est dire l'enjeu de ces transferts.

La cinquième partie, "Tradition, innovation, adaptation », souffre un peu des « limites » - si l'on ose dire - de l'entreprise : l'influence de la production jésuite dans le domaine de la théorie politique reste mal représentée par un article d'Irving Lavin sur le prince chrétien. En revanche, dans le domaine, à nouveau, de l'histoire des sciences, l'enquête de Markus Hellyer sur l'enseignement de la physique dans les collèges allemands dégage d'importantes perspectives sur le rôle de la censure dans la négociation des ouvertures de la tradition à l'innovation ${ }^{12}$.

La dernière partie de l'ouvrage, dont l'objet semble hésiter entre la « dévotion », peu abordée dans l'ensemble, et de nouveaux développements sur les arts, bénéficie cependant d'un intéressant article de James F. Keenan sur les rapports de la « spiritualité » jésuite et du «puritanisme » anglican. Il montre comment le processus de la « conversion » intérieure des formes extérieures de la dévotion et du culte religieux déplace les frontières, et met en évidence la richesse du « terrain » anglais, peu exploré de ce point de vue ${ }^{13}$.

Pour finir, est-il possible d'avancer une hypothèse ironique? Le succès, dont témoignent plusieurs moments de l'ouvrage, de la définition, par Marc Fumaroli, de l'Imago Primi Saeculi, composé en 1640 pour la célébration du premier siècle de la Compagnie de Jésus, comme " architecture-livre ", pourrait bien être l'effet du « refoulement » relatif de l'institution dans l'ensemble du volume. Mais, du coup, ce sont les marges de l'édifice, telles qu'elles apparaissent, par exemple, dans les jardins sauvages d'une autre architecture-livre, celle de la Peinture spirituelle de Louis Richeome (1611), qui se trouvent, elles aussi, refoulées, celles qui apparầtraient aux limites d'une histoire internaliste et d'une histoire externaliste de l'institution.

Pierre-Antoine FABRE (avril 1999).

11. Renvoyons aussi, dans la même direction, au doctorat de Pascale Girard sur les franciscains de Chine, cité dans l'introduction de ce numéro, supra, p. 254, en particulier pour l'étude du Dialogue de Pedro de Piñuela (1686).

12. Ce chantier de recherches sera certainement l'un des principaux des prochaines années, à la faveur, aussi, de l'ouverture des archives du Saint-Office: voir sur ce point l'article d'Antonella Romano, «Pratiques d'enseignement et orthodoxie intellectuelle en milieu jésuite (seconde moité du xvı siècle) ", in Orthodoxie, christianisme, histoire. Travaux du groupe de recherche «Définir, maintenir et remettre en question l'orthodoxie dans l'histoire du christianisme ", éd. Éric ReBILlard et Antonella Romano, Rome, École française de Rome (Collection de l'École française de Rome), sous presse.

13. Signalons toutefois, sur le sujet de ces fronts renversés, l'étude récente de Jean-Pierre CAvallú sur les stratégies de résistance des jésuites anglais à la répression, «Équivoques et restrictions mentales ", Kairos, 8, 1997, p. 35-80. 